\title{
Anatomie Et Histologie De L'hepatopancreas Chez Le Male Du Poisson Brachydeuterus Auritus (Haemulidae, Valenciennes, 1831) En Cote D'ivoire
}

\author{
Komenan Daouda Kouassi \\ Marie-Anne D'Almeida \\ Jean Jacques Miessan
}

Laboratoire de Biologie Cellulaire, UFR Biosciences-Université Félix Houphouët Boigny, Abidjan, Côte d'Ivoire

Silvain Yao

Valentin N'Douba

Laboratoire d'Hydrobiologie, UFR Biosciences-Université Félix Houphouët

Boigny, Abidjan, Côte d'Ivoire

\section{Goueh Gnahoue}

Laboratoire de Biochimie et de Microbiologie, Ecole Normale Supérieure

(ENS), Abidjan, Côte d'Ivoire

doi: 10.19044/esj.2017.v13n24p289 URL:http://dx.doi.org/10.19044/esj.2017.v13n24p289

\begin{abstract}
Brachydeuterus auritus is a fish species with a high level of exploitation potential in Côte d'ivoire. The following study is based upon a maturity ladder and the discrimination of the hepatopancreas. The methodological approach consisted of a macroscopic description of the hepatopancreas according to the five stages of sexual maturity. The microscopic study consisted of fixing the hepatopancreas, dehydrating, impregnating, including it, making cuts that are colored for observation.

At the macroscopic level, the hepatopancreas is made of two unequal lobes. The organ has a colouring as well as a vascularization varying in terms of the species development stages. At the microscopic level among the males, the hepatopancreas is a mixed organ made of the liver and the pancreas. The hepatopancreatic parenchyma is an homogeneous tissue made of hematocytes which are polygonal cells. In addition, we can find hematopoietic tissues in the parenchyma and melanomacrophages centers located near the blood vessels and the biliary tracts. The melanomacrophages centers are formed with cells accumulating pigment cells. Inside the organ, the pancreas is discriminated centripally and constituted of exocrine cells and Langerhans islets. The physiology of the two entities (liver and
\end{abstract}


pancreas) will establish the organ's physiology. Hepatopancreas is involved in reproduction, digestion and defence. It exists as an organ that controls many functions and plays an important part in the physiology of fish in anabolism and catabolism.

Keywords: Brachydeuterus auritus; male ; hepatopancreas ; anatomy ; histology

\section{Resume}

Brachydeuterus auritus est une espèce de poisson qui présente un fort potentiel d'exploitation en Côte d'Ivoire. La présente étude porte sur l'échelle de maturité et la différenciation de l'hépatopancréas. La démarche méthodologique a consisté à une description macroscopique de l'hépatopancréas en fonction des cinq stades de maturité sexuelle. L'étude microscopique a consisté à fixer les hépatopancréas, à les déshydrater, à les imprégner, à les inclure, à réaliser les coupes qui seront colorées en vue d'une observation. Au plan macroscopique, l'hépatopancréas est constitué de deux lobes inégaux. L'organe présente une coloration, des granulations et une vascularisation qui varient en fonction des stades de développement de l'espèce. Au plan microscopique, chez le mâle, l'hépatopancréas est un organe mixte constitué du foie et du pancréas. Le parenchyme hépatique est un tissu homogène, formé d'hépatocytes qui sont des cellules polygonales. En outre, on distingue dans le parenchyme des tissus hématopoïétiques et des centres mélanomacrophages, répartis près des vaisseaux sanguins et des voies biliaires. Les centres mélanomacrophages sont constitués de cellules accumulant des pigments. Au sein de l'organe, le pancréas se différencie de façon centripète. Il est constitué de cellules exocrines et des îlots de Langerhans. La physiologie des deux entités à savoir le foie et le pancréas détermine la physiologie de l'organe. L'hépatopancréas intervient dans la reproduction, la digestion et la défense. Il apparaît comme un organe qui contrôle de nombreuses fonctions et joue un rôle important dans la physiologie des poissons tant dans l'anabolisme que dans le catabolisme.

Mots clés: Brachydeuterus auritus; mâle; hépatopancréas ; anatomie ; histologie

\section{Introduction}

En Côte d'Ivoire, le poisson tient une place importante dans l'alimentation. Il représente la seule forme de protéines animales accessibles et/ou ayant un prix abordable pour les ménages en milieu urbain et périurbain en Afrique (Chilima, 2008). Sur le marché ivoirien, parmi les espèces prisées compte tenu de leur très haute valeur culinaire se trouvent Brachydeuterus auritus de la famille des Haemulidae. 
Dans la littérature, plusieurs travaux se rapportent aux espèces pélagiques et benthiques. La majorité de ces espèces appartiennent au plateau continental ivoirien. Parmi ces espèces marines, on distingue Brachydeuterus auritus (Valencienne, 1831) une espèce pélagique. Exploités depuis plusieurs décennies, la demande nationale en poissons en particulier Brachydeuterus auritus reste forte et soutenue. Cette forte demande sur les marchés induit une pêche intensive. Pour faire face à une éventuelle pénurie, la maîtrise de leur biologie entre autre de leur cycle reproductif est primordiale pour envisager une pisciculture ultérieure.

Bien que présentant des intérêts nutritionnels, économiques appréciables en côte d'Ivoire, cette espèce n'a fait l'objet d'aucune étude. Leur cycle sexuel et leur reproduction sont méconnus. En effet, les cycles de reproduction impliquent un ensemble de processus physiologiques et comportementaux en rapport avec divers facteurs de l'environnement biotique et abiotique (Paugy et Levêque, 1999). L'effort de reproduction occasionne d'importantes dépenses énergétiques soutenues par l'apport direct de nutriments mais surtout par l'utilisation de réserves préalablement constituées et stockées dans l'hépatopancréas. La maîtrise de la biologie de Brachydeuterus auritus passe également par la connaissance de leur cycle et celle de son organe de stockage énergétique, l'hépatopancréas nécessaire à sa gamétogénèse. La différenciation de 1'hépatopancréas et des gonades a lieu durant la maturité sexuelle de Brachydeuterus auritus. Ce présent travail veut contribuer à l'acquisition des connaissances sur l'hépatopancréas des Poissons. Il s'agit de faire l'étude de l'échelle de maturité et de la différenciation de l'hépatopancréas au plan macroscopique et microscopique chez les mâles de Brachydeuterus auritus.

\section{Matériel et méthode}

\section{Matériel biologique}

L'étude a porté sur 118 spécimens de Brachydeuterus auritus. L'échantillonnage a été effectué au port de pêche d'Abidjan de Janvier 2016 à Janvier 2017. Certains échantillons ont été disséqués sur place. D'autres ont été disséqués après un temps de congélation. Tous les hépatopancréas ont été prélevés pour l'étude histologique.

\section{Méthode macroscopique}

L'approvisionnement s'est fait au port autonome d'Abidjan. Les poissons ont été conservés dans une glacière contenant de la glace afin d'éviter l'altération des différents tissus à étudier. Convoyés au laboratoire, ces poissons ont été transférés au congélateur pendant une heure avant les différentes manipulations qui comporte deux volets macroscopique et microscopique. 
Au plan macroscopique les critères retenus :

L'observation morphologique et les différentes mesures.

Les paramètres mesurés sont la taille des poissons et de l'hépatopancréas grâce à une règle graduée et le poids grâce à la balance de marque Tefal optiss de capacité $5 \mathrm{Kg}$. Concernant la taille les mesures prises sur le spécimen sont:

- La longueur totale (Lt) en cm, qui est mesurée de l'extrémité du museau à celle de l'extrémité extrême d'une des nageoires caudales.

- La hauteur en $\mathrm{cm}$.

Les mesures prises sur l'hépatopancréas sont :

La longueur de l'hépatopancréas.

Quant aux poids, les mesures suivantes ont été prises sur les specimen :

- La masse $(\mathrm{Mt})$ au gramme près du poisson non éviscéré.

Les poids des hépatopancréas ont été également déterminés :

- La masse (Mh) au de gramme près des deux lobes de l'hépatopancréas des mâles et des femelles.

L'étude de l'hépatopancréas est corrélée avec l'échelle de maturité car sa différenciation se fait selon cette maturité. Bien que l'espèce soit gonochorique il n'existe pas de dimorphisme sexuel chez ces poissons. Il ne présente aucun critère externe pour identifier les mâles et femelles. C'est l'observation de la différenciation des gonades qui a permis de classer les individus selon les sexes et les stades de maturité. Cette observation n'a été possible qu'après dissection.

\section{Méthode technique}

Les techniques histologiques de référence de Martoja et MartojaPierson (1967) ont été appliquées. L'étude histologique n'a concerné que l'hépatopancréas des poissons aux différents stades de maturité. Les échantillons ont été prélevés et fixés par immersion dans du liquide de Bouin aqueux ou dans du formol à $10 \%$, déshydratés de façon progressive à l'éthanol $\left(70^{\circ}, 95^{\circ}\right.$ et $\left.100^{\circ}\right)$ puis préimprègnés dans du butanol pour les échantillons fixés au Bouin et dans du toluène pour les échantillons fixés dans le formol à $10 \%$. L'imprégnation proprement dite ainsi que l'inclusion ont été faites dans de la paraffine (PARAFINA PARA HISTOLOGICA). Des coupes de $7 \mu \mathrm{m}$ réalisées au microtome MICROM, ont été colorées à l'hémalun-éosine. Pour obtenir des coupes convenables sans déchirure, les blocs histologiques entamés ont été constamment trempés 12 à $24 \mathrm{~h}$ dans un bac contenant de l'eau courante. Ce trempage permet la réalisation aisée des coupes. Cette opération est répétée chaque fois que les blocs s'effritent pendant l'étape de la microtomie. Les coupes ont également été collodionnées pour éviter leur décollement. L'observation et les photos ont été faites au photomicroscope Motic et OLYMPUSCKX41. 


\section{Résultats}

\section{Echelle de maturité sexuelle}

Les manipulations ont concerné l'observation des caractéristiques externes et internes des individus car il n'existe pas de dimorphisme sexuel chez ces poissons. L'observation de l'anatomie interne, précisément la différenciation des gonades a servi de base pour l'établissement de l'échelle de maturité. L'hépatopancréas a été prélevé selon ces différents stades. Les mâles ont été identifiés après dissection et observation des gonades dont la taille et la couleur varient du stade juvénile au stade adulte. L'échelle de maturité comporte cinq stades.

Aux stades juvéniles I et II, la couleur des testicules est blanchâtre. Ils sont filiformes au stade I et peu développé au stade II.

Au stade III et IV les gonades sont blanches et développées.

$\mathrm{Au}$ stade $\mathrm{V}$, les gonades sont flasques.

\section{Différenciation macroscopique de l'hépatopancréas chez les mâles de Brachydeuterus auritus}

Les hépatopancréas des mâles chez Brachydeuterus auritus présentent cinq stades de maturité (figure 1).

- Stade 1: les individus mesurent en moyenne $13.4 \mathrm{~cm}$ avec une hauteur moyenne de $3.6 \mathrm{~cm}$ et une masse de $23 \mathrm{~g}$ (figure $1 \mathrm{~A}$ ). Leurs hépatopancréas mesurent $2.7 \mathrm{~cm}$. Ils pèsent $0.7 \mathrm{~g}$ et sont formés de deux lobes inégaux de couleur marron. Le lobe gauche est développé que le lobe droit (figure $1 \mathrm{~A} 1$ ). L'organe hépatopancréatique renferme des granules de $0.18 \mathrm{~mm}$ de diamètre. Les vaisseaux sanguins sont de faible calibre (figure $2 \mathrm{~A}$ ).

- Stade 2: il regroupe les spécimens qui pèsent $60 \mathrm{~g}$ et dont la taille moyenne est $14.7 \mathrm{~cm}$ sur $3.8 \mathrm{~cm}$ de hauteur (figure 1B). Ils possèdent des hépatopancréas mesurant en moyenne $3.1 \mathrm{~cm}$. Ces hépatopancréas de 0.8 $\mathrm{g}$ sont de couleur marron avec deux lobes qui ont presque la même taille mais le lobe gauche demeure plus volumineux que le lobe droit (figure 1 $\mathrm{B} 1)$. Le diamètre granulaire est de $0.18 \mathrm{~mm}$. Les vaisseaux sanguins de faible calibre se ramifient (figure $2 \mathrm{~B}$ ).

- Stade 3: les individus ont une masse de $86 \mathrm{~g}$. Leur longueur totale moyenne est de $15.5 \mathrm{~cm}$ et leur hauteur moyenne est de $4 \mathrm{~cm}$ (figure 1C). Ils sont dotés d'hépatopancréas longs de $3.3 \mathrm{~cm}$ de masse égale à $0.9 \mathrm{~g}$. Les organes hépatopancréatiques sont bilobés et de couleur marron. Les deux lobes sont de taille égale mais le lobe gauche est plus volumineux que le lobe droit (figure 1C1). Les granules de $0.19 \mathrm{~mm}$ de diamètre sont réunis en grappes. Leurs contours sont distincts et sont parsemés de vaisseaux sanguins de calibre plus élevé qu'au stade précédent (figure 2C). 
- Stade $4:$ la taille des spécimens atteint $17.6 \mathrm{~cm}$ et la hauteur vaut $4.7 \mathrm{~cm}$. Ils pèsent $109 \mathrm{~g}$ (figure 1D). Ils possèdent des hépatopancréas de $0.9 \mathrm{~g}$, de couleur marron clair et bilobulaires qui mesurent $4.1 \mathrm{~cm}$. Les lobes sont de taille égale, cependant le lobe gauche est légèrement plus volumineux que le lobe droit (figure 1D1). Le diamètre des granules est de $0.19 \mathrm{~mm}$. Ils sont bien délimités par leur contour et disposés en agrégats (figure 2D).

- Stade 5 : les spécimens de $113 \mathrm{~g}$, ont une taille de $17.1 \mathrm{~cm}$ et une hauteur de $4.5 \mathrm{~cm}$ (figure 1E). Leurs hépatopancréas pèsent $1 \mathrm{~g}$. Ils sont longs de $3.7 \mathrm{~cm}$ et de couleur violet. Ils sont constitués de deux lobes presque proportionnels (figure 1E1). Les vaisseaux sanguins ont légèrement augmenté de volume et le diamètre granulaire équivaut à $0.19 \mathrm{~mm}$ (figure 2E).

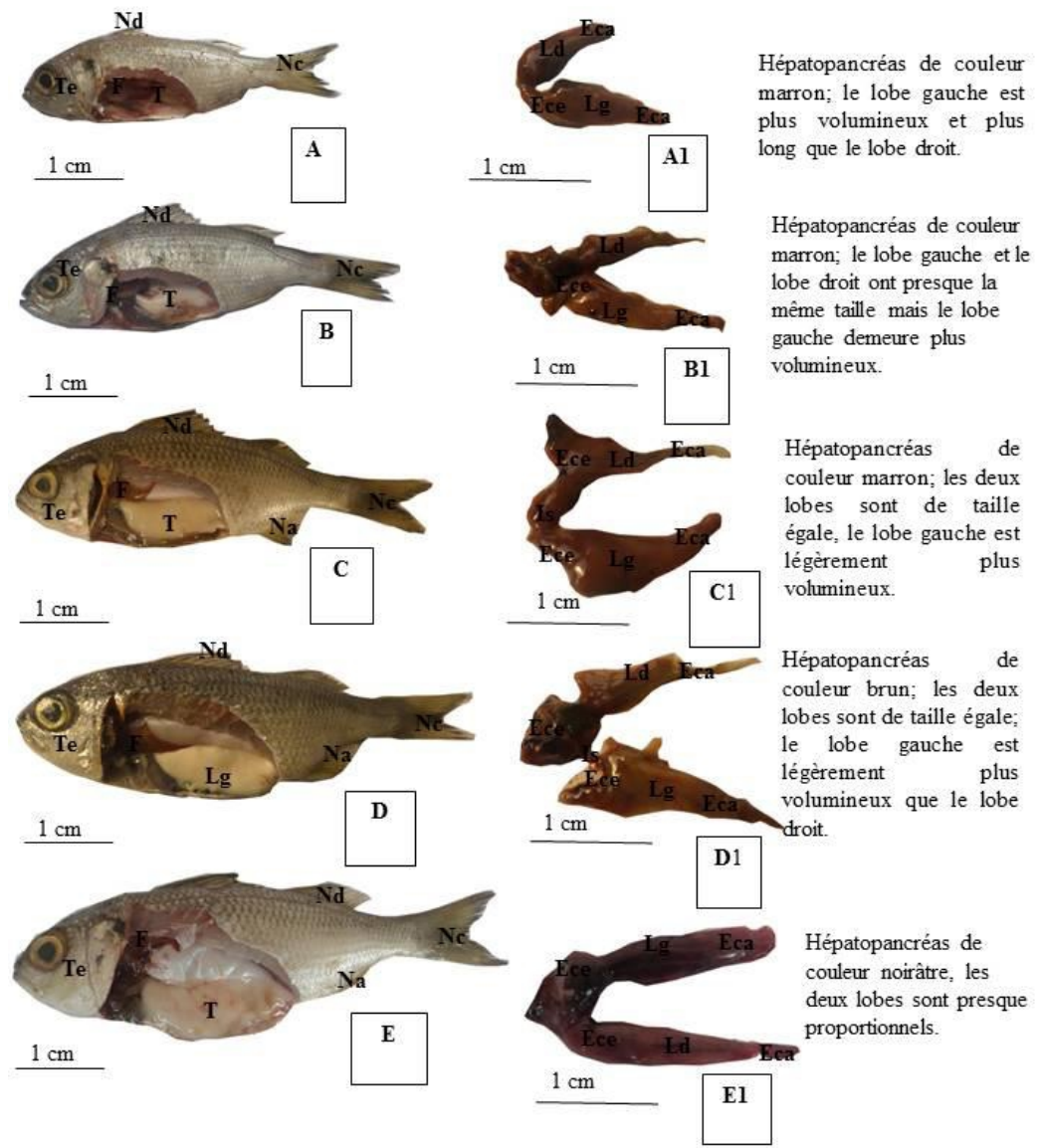

Figure 1: Echelle de maturité et de différenciation de l'hépatopancréas chez le mâle de Brachydeuterus auritus:

A: juvénile mâle au stade $1 ; \mathbf{A l}$ : hépatopancréas du mâle au stade1; $\mathbf{B}$ : juvénile mâle au stade 2 ; B1: hépatopancréas du mâle au stade 2; $\mathbf{C}$ : mâle adulte au stade 3; Cl: hépatopancréas au stade 3; D: mâle adulte au stade 4; D1: hépatopancréas au stade $4 ; \mathbf{E}$ : mâle adulte au stade5; E1 hépatopancréas au stade 5; Nc: nageoire caudale; Nd: nageoire dorsale; Na: nageoire anale; Te: tête; T: testicule; Ffoie; Ece: extrémité céphalique du foie; Eca: extrémité caudale du foie; Ld: lobe droit; Lg: lobe gauche; Is: Isthme 

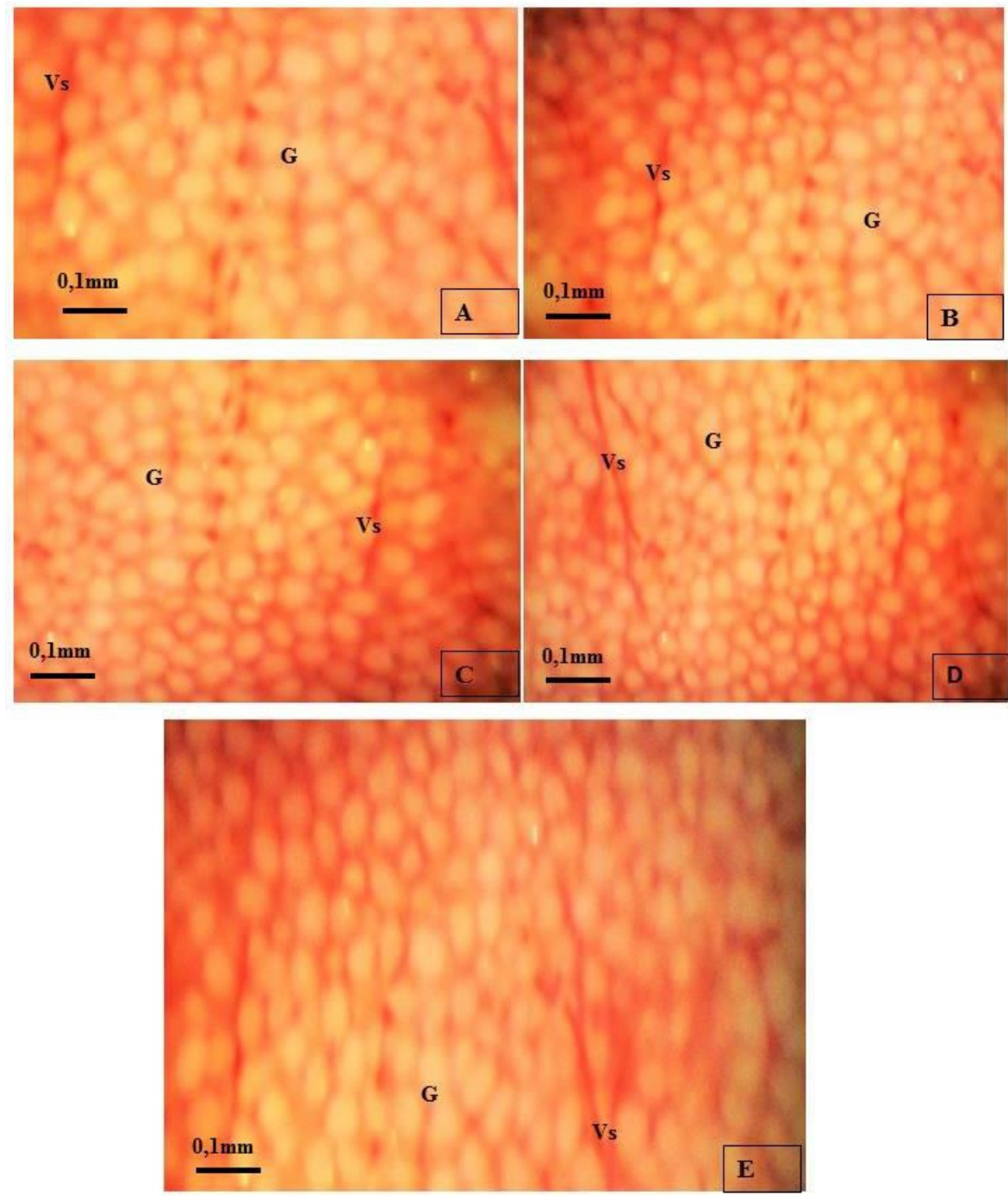

Figure 2: Evolution de la granulation et de la vascularisation des hépatopancréas chez les mâles de Brachydeuterus auritus à différents stades de maturité: $\mathbf{A}$ : stade $1 ; \mathbf{B}$ stade:2; $\mathbf{C}$ : stade:3; D: stade 4; E: stade 5; $\mathbf{G}$; granule; Vs: vaisseau sanguin

Différenciation microscopique de l'hépatopancréas chez les mâles de Brachydeuterus auritus

- Stade 1

L'hépatopancréas au stade 1 (figure 3A) est constitué du parenchyme hépatique et du pancréas. Le parenchyme hépatique est essentiellement constitué de cellules hépatiques ou hépatocytes de forme polygonale, rassemblées en travées autour de sinusoïdes (capillaires) qui contiennent des érythrocytes. Il est parsemé de capillaires sanguins : veinules et de vaisseaux sanguins : les veines et les artères (figures $3 \mathrm{C}$ et $3 \mathrm{E}$ ). Les capillaires sanguins 
sont de faible calibre. Les artères ont une épaisse paroi et un calibre moyen tandis que les veines présentent un gros calibre mais une paroi mince. On distingue dans le parenchyme, les canaux biliaires disposés à proximité du tractus veino-pancréatique (figure 3D). Quant au pancréas, il est constitué de deux parties (figure 3D): le pancréas endocrine et le pancréas exocrine. Il est disséminé autour des branches de veine porte dans le parenchyme hépatique et présente une bifurcation. Le pancréas endocrine est représenté par les îlots de Langerhans non visible sur la photographie et le pancréas exocrine est constitué des acini séreux pancréatiques.

- $\quad$ Stade 2

L'hépatopancréas (figure 4A) présente un parenchyme hépatique constitué d'hépatocytes de taille supérieure à celle du stade 1et de vaisseaux sanguins, des sinusoïdes, des canaux biliaires et du tractus veinopancréatiques dont le calibre a légèrement augmenté (figures $4 B, C, D$, E et F). Le tractus veino-pancréatique est constitué de branches de veine porte, du pancréas exocrine et du pancréas endocrine (figure $4 \mathrm{D}$ ). La section longitudinale du canal biliaire montre le passage de Héring (figure $4 \mathrm{~F}$ ).

- Stade 3

L'hépatopancréas (figure $5 \mathrm{~A}$ ) présente des hépatocytes dévéloppés qui laissent dans leurs travées les sinusoïdes gorgées d'érythrocytes (figures $5 \mathrm{C}$ et $5 \mathrm{E}$ ). Les cellules mélanomacrophages sont présentes (figure $5 \mathrm{~F}$ ).

- $\quad$ Stade 4

L'observation microscopique de l'hépatopancréas à ce stade (figure 6 A) montre la présence des hépatocytes très développés. Une triade constituée d'une artère, d'une veine et d'un canal biliaire est observée (figures $6 \mathrm{C}$ et $6 \mathrm{E}$ ). La bifurcation du pancréas débute vers une extrémité (figure $6 \mathrm{~F}$ ) et progresse vers l'intérieur de l'organe (figure 6D). La différenciation des cellules pancréatiques se fait donc par bifurcation centripète (figures $6 \mathrm{D}$ et $6 \mathrm{~F})$.

- Stade 5

La coupe histologique de l'hépatopancréas (figure 7A) montre des hépatocytes très développés (figures $7 \mathrm{C}$ et $7 \mathrm{D}$ ). La veine se ramifie en veinules (figure 7D). Un centre mélanomacrophage est localisé près d'une artère et d'une veinule (figure 7E). 

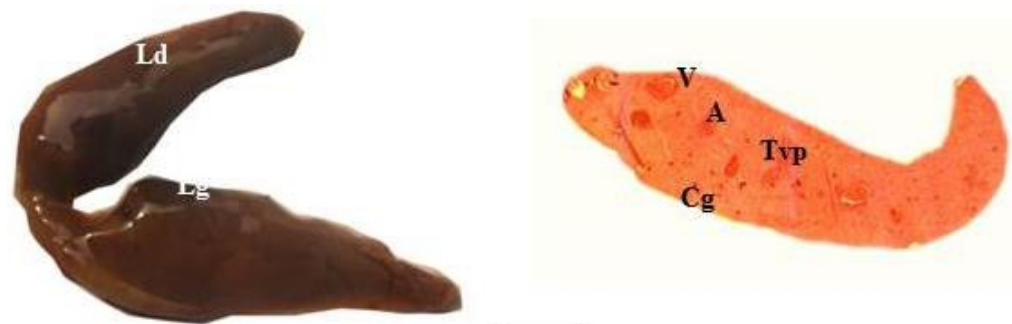

\section{$\mathbf{A}$}
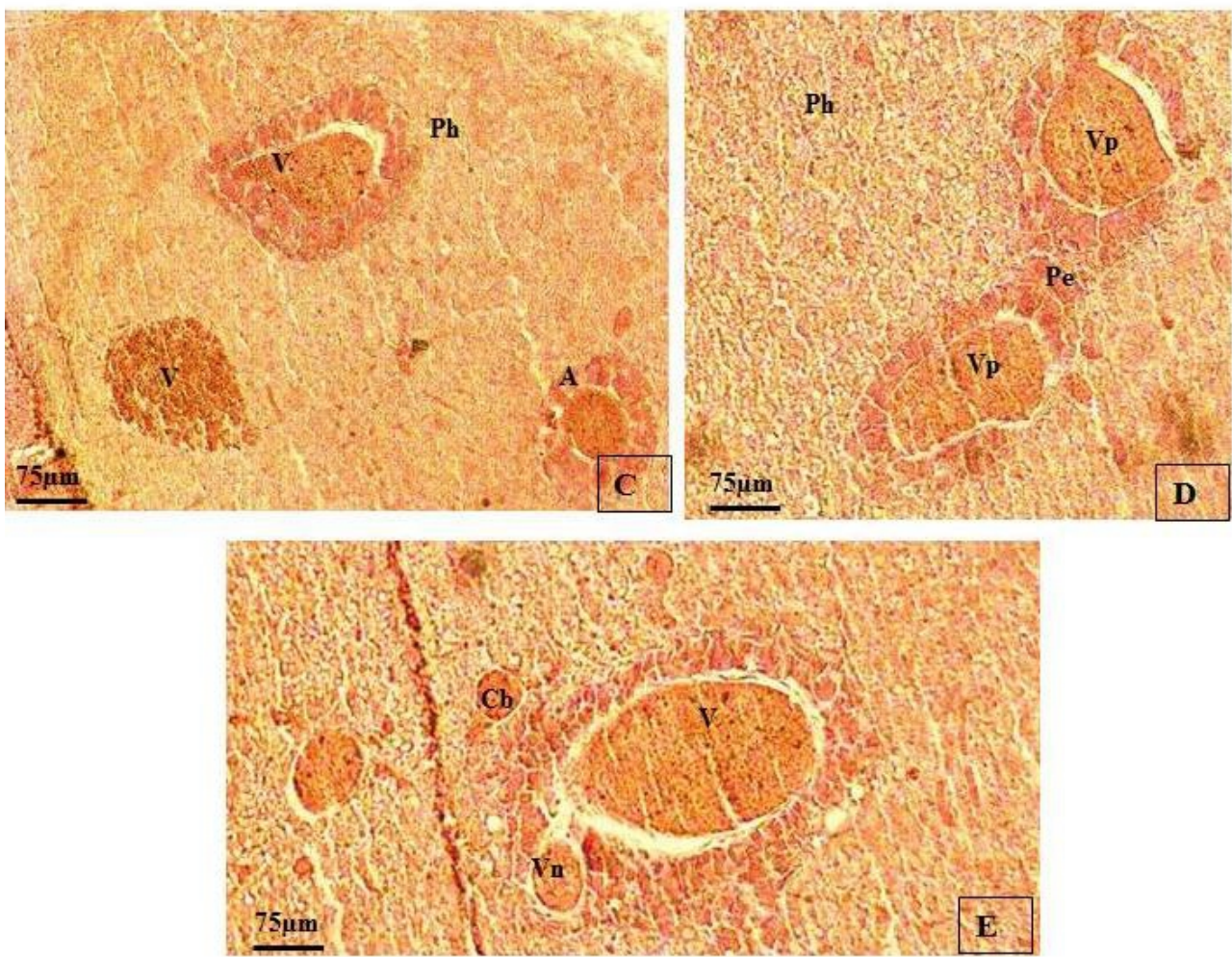

Figure 3: Anatomie et histologie de l'hépatopancréas du mâle de Brachydeuterus auritus au stade 1: A: anatomie de l'hépatopancréas; B: vue d'ensemble d'une portion de la coupe de l'hépatopancréas; $\mathbf{C}$, $\mathbf{D}, \mathbf{E}$ : vues de portions détaillées de l'hépatopancréas; $\mathbf{A}$ : artère; $\mathbf{V}$ : veine; $\mathbf{V n}$ : veinule; $\mathbf{C b}$ : canal biliaire; $\mathbf{C g}$ : capsule de Glisson; Vp: veine porte; Ph: parenchyme hépatique; Tvp: tractus veino-pancréatique; Lg: lobe gauche; Ld: lobe droit

Coloration: hématoxyline-éosine

B: $\mathbf{G ~ X ~} 40$

C, D, E: G X 100 

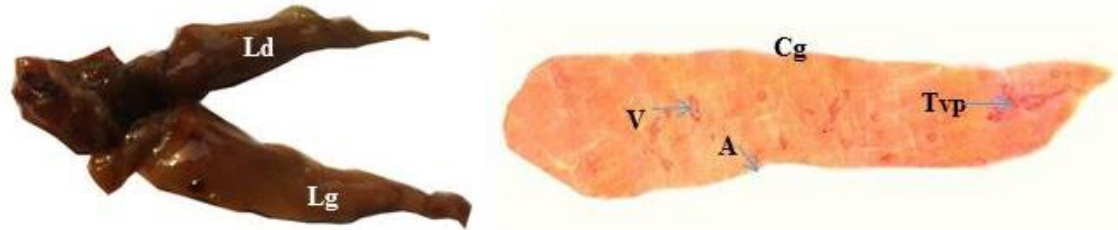

A
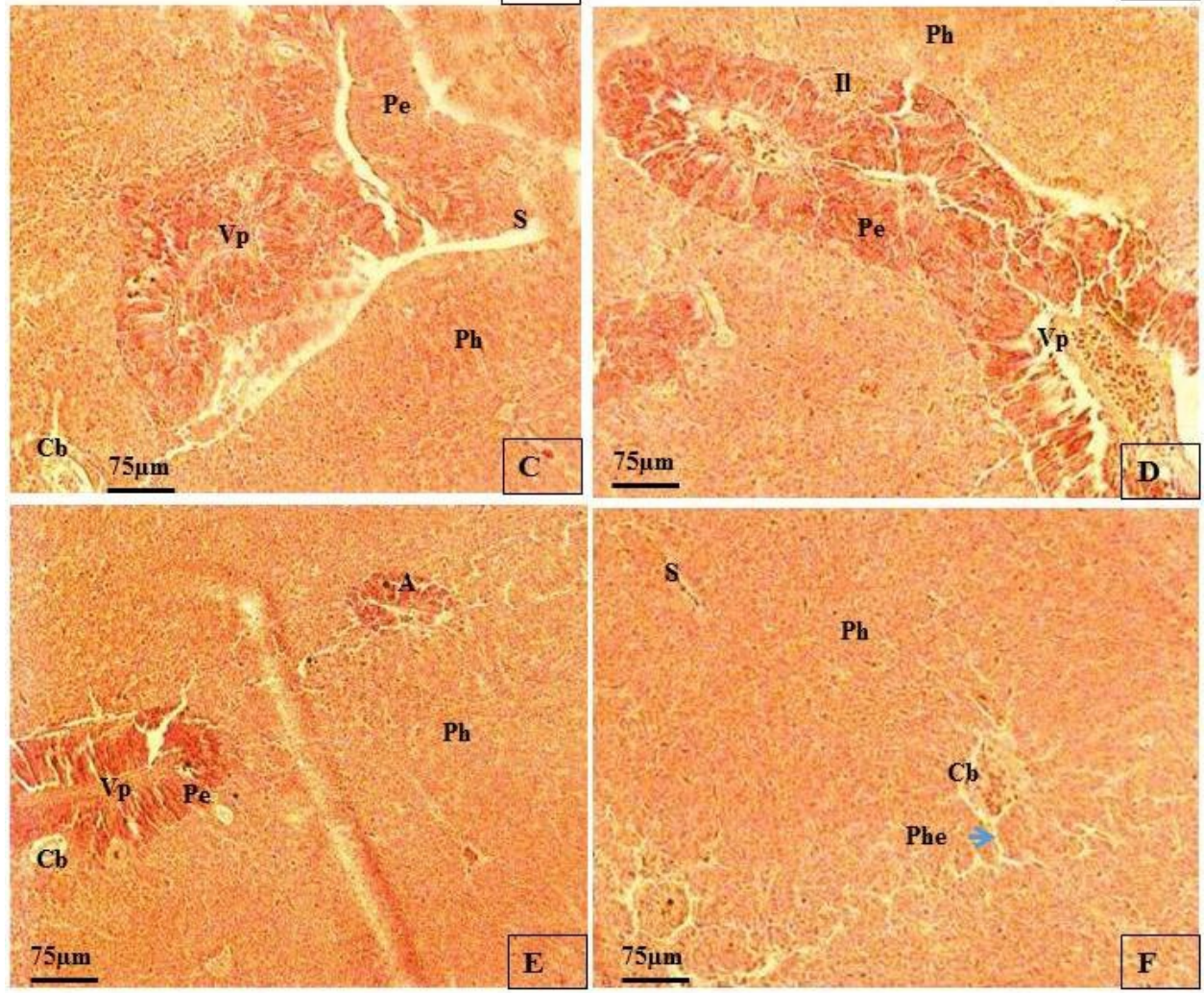

Figure 4: Anatomie et histologie de l'hépatopancréas du mâle de Brachydeuterus auritus au stade 2; A: anatomie de l'hépatopancréas; $\mathbf{B}$ : vue d'ensemble d'une portion de la coupe de l'hépatopancréas; $\mathbf{C}, \mathbf{D}$, $\mathbf{E}, \mathbf{F}$ : vues détaillées de portions de la coupe de l'hépatopancréas; $\mathbf{A}$ : artère; V: veine; $\mathbf{S}$ : sinusoide; $\mathbf{C b}$ : canal biliaire; Ph: parenchyme hépatique; $\mathbf{C g}$ : capsule de Glisson; Phe: passage de Héring; Il: îlot de Langerhans; Tvp: tractus veino-pancréatique; Vp: veine porte; Ld: lobe droit; Lg: lobe gauche

Coloration: hématoxyline-éosine

B: $\mathbf{G X} \mathbf{4 0}$

C, D, E, F: G X 100 

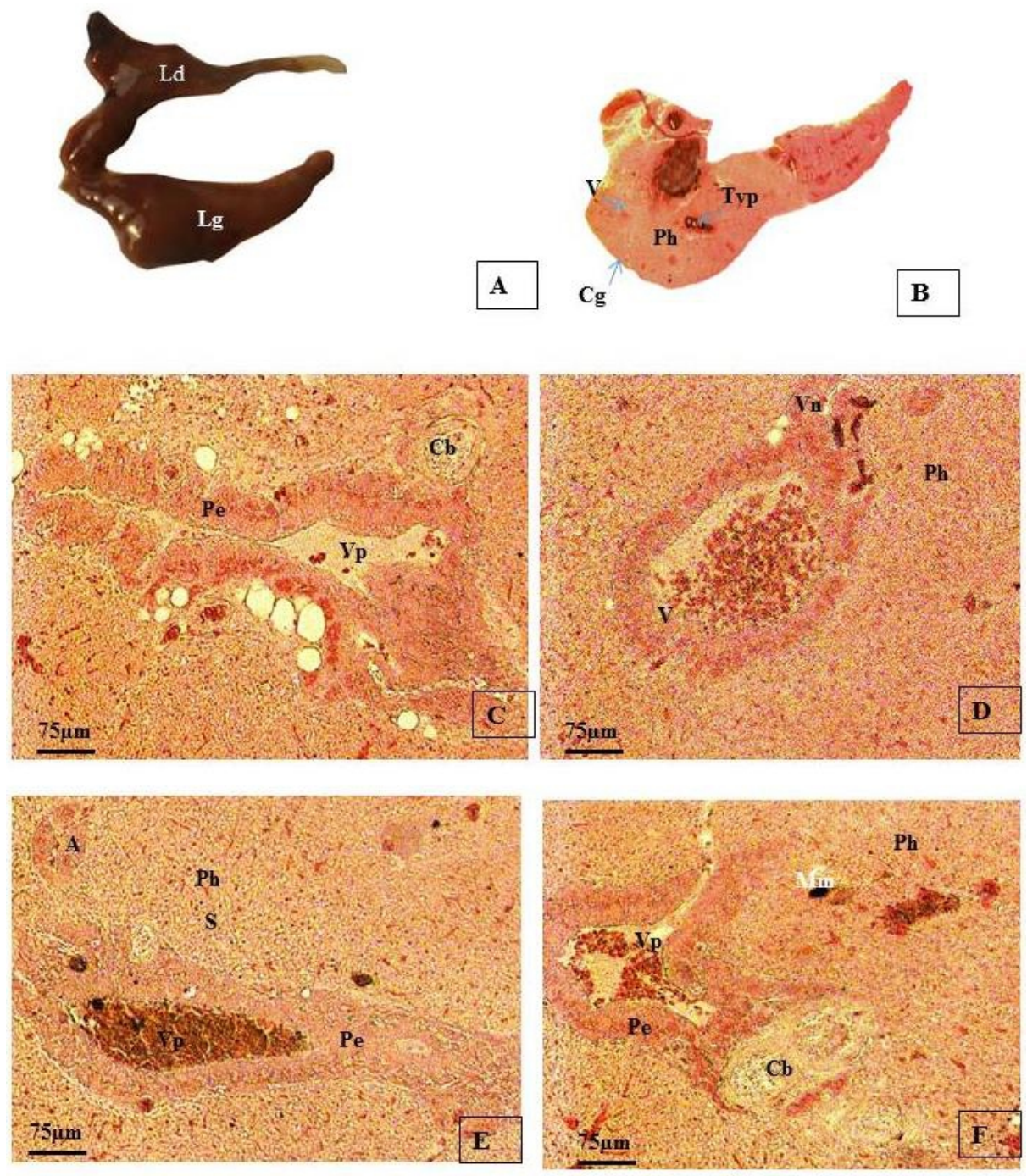

Figure 5: Anatomie et histologie de l'hépatopancréas du mâle de Brachydeuterus auritus au stade 3: A: anatomie de l'hépatopancréas; $\mathbf{B}$ : vue d'ensemble d'une portion de la coupe de l'hépatopancréas; $\mathbf{C}$, D, E, F: vues détaillées de portions de la coupe de l'hépatopancréas; $\mathbf{C g}$ : capsule de Glisson; V: veine; A:artère; Vp: veine porte; Pe: pancréas exocrine; Vn: veinule; $\mathbf{M m}$ : mélanomacrophage; $\mathbf{S}$ : sinusoīde; $\mathbf{C b}$ : canal biliaire; $\mathbf{L g}$ : lobe gauche; $\mathbf{L d}$ : lobe droit; $\mathbf{P h}$ : parenchyme hépatique; $\mathbf{T v p}$ : tractus veinopancréatique

Coloration: hématoxyline-éosine

B: $\mathbf{G X} 40$

C, D, E, F: GX 100 


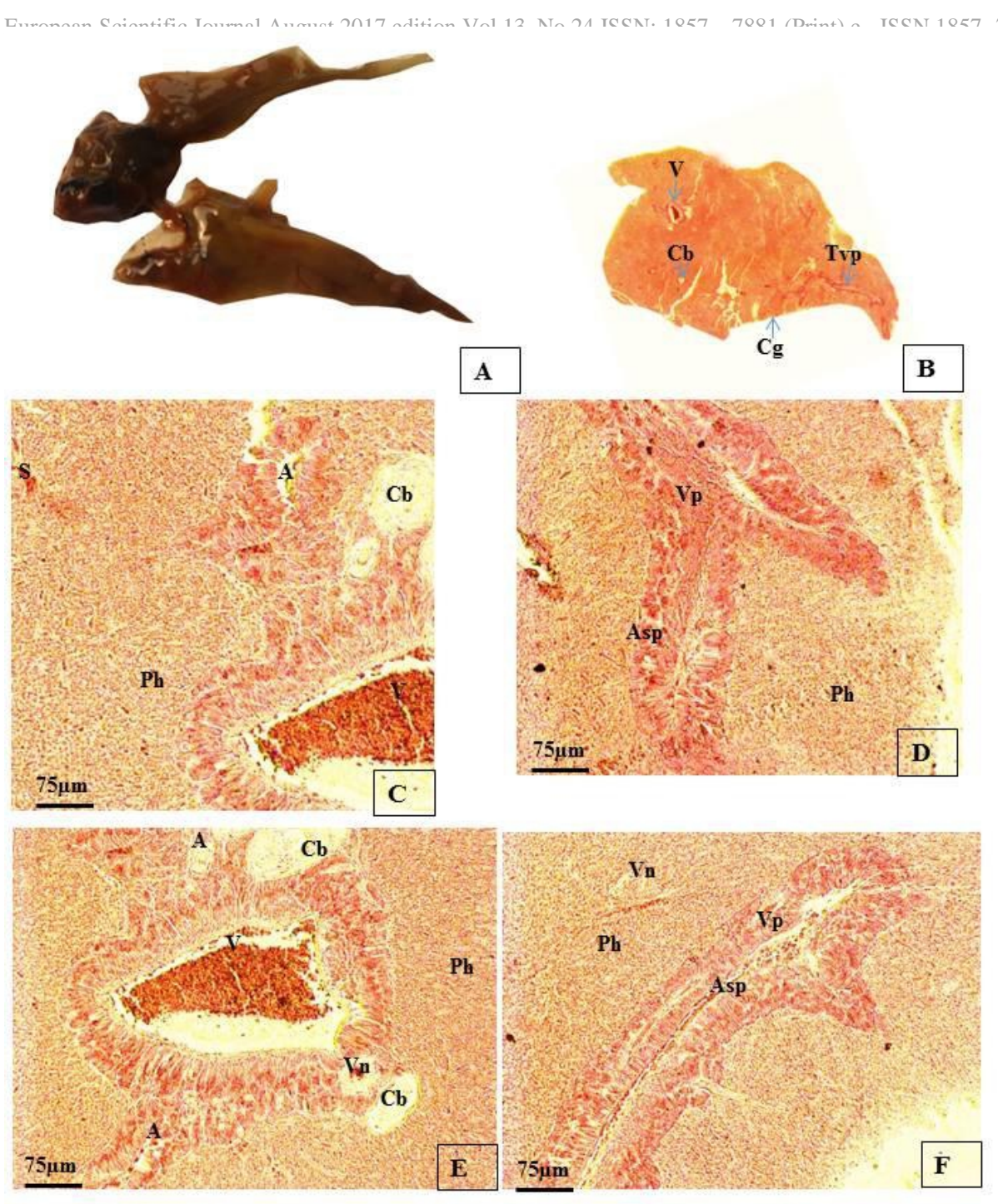

Figure 6: Anatomie et histologie de l'hépatopancréas du mâle de Brachydeuterus auritus au stade 4: A: anatomie de l'hépatopancréas; $\mathbf{B}$ : vue générale d'une portion de coupe de l'hépatopancréas; $\mathbf{C}, \mathbf{D}$, $\mathbf{E}, \mathbf{F}$ : vues détaillées de portions de l'hépatopancréas; $\mathbf{A}$ : artère; $\mathbf{V}$ : veine; $\mathbf{C b}$ : canal biliaire; Vn:veinule; Ph: parenchyme hépatique; Asp: Acini séreux pancréatiques; Vp: veine porte; S: sinusoide; Tvp: tractus veino-pancréatique; $\mathbf{C g}$ : capsule de Glisson; $\mathbf{L g}$ : lobe gauche; Ld: lobe droit Coloration:hêma toxyline-éosine

B: $\mathbf{G ~ X ~} \mathbf{4 0}$

C, D, E, F: G X 100 


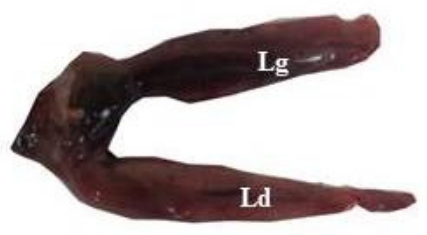

A

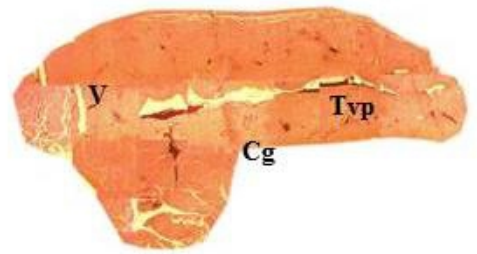

B
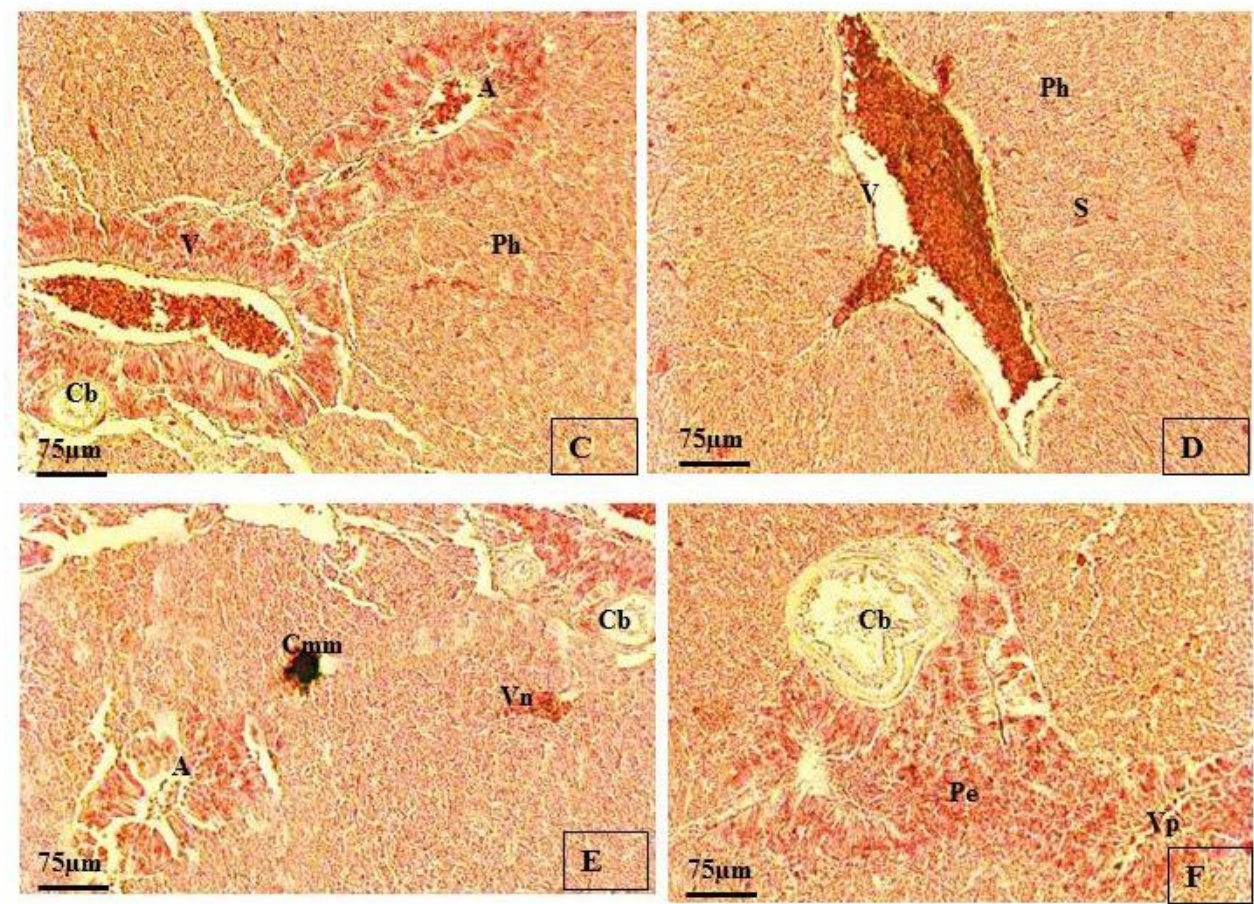

Figure 7: Anatomie et histologie de l'hépatopancréas du mâle de Brachydeuterus auritus au stade 5: A: anatomie de l'hépatopancréas; $B$ : vue d'ensemble d'une portion de la coupe de l'hépatopancréas; C, D, E, F: vues détaillées de portions de 1'hépatopancréas; A: artère; V: veine; Pe: pancréas exocrine; S: sinusoĩde; $\mathbf{P h}$ : parenchyme hépatique; $\mathbf{C g}$ : capsule de Glisson; Vp: veine porte; Tvp: tractus veinopancréatique; $\mathbf{L g}$ : lobe gauche; Ld: lobe droit; $\mathbf{C m m}$ : centre mélanomacrophage

Coloration: hématoxyline-éosine

B: $\mathbf{G ~ X ~} 40$

C, D, E, F: G X 100 


\section{Discussion}

La maturité de l'hépatopancréas se fait suivant 5 stades chez les mâles de Brachydeuterus auritus. Il ressort de cette étude que tous les individus dont la longueur de l'hépatopancréas est comprise entre $2.7 \mathrm{~cm}$ et $3.1 \mathrm{~cm}$ sont immatures. Ce groupe correspond aux individus des stades $1 \mathrm{et}$ 2. Les mâles pubères correspondant au stade 3 ont un hépatopancréas dont la longueur équivaut à $3.3 \mathrm{~cm}$. Les individus mâtures du stade 4 et du stade 5 ont leur hépatopancréas qui mesure entre $4.1 \mathrm{~cm}$ et $3.7 \mathrm{~cm}$. Faute de références bibliographiques relatives à l'échelle de maturité de l'hépatopancréas chez les poissons et autres classes d'animaux, aucune comparaison n'a été possible.

L'étude macroscopique des hépatopancréas des mâles de Brachydeuterus auritus a montré qu'ils sont tous pourvus de deux lobes généralement inégaux. Ces résultats corroborent ceux de Claudemir et al. (2014) qui ont montré que le foie de Hemisorubim platyrhynchos possède deux lobes inégaux. La morphologie de l'hépatopancréas varie en fonction des stades de maturité voire au sein du même stade. Ce polymorphisme de l'hépatopancréas a été noté par Genten et al. (2011). Pendant la maturité de l'hépatopancréas, sa taille croît du stade 1 au stade 5 pendant que sa vascularisation s'intensifie. De même la forme et le poids augmentent du stade 1 au stade 5. Ceci confirme les résultats de Bertolucci et al.( 2008) . L'hépatopancréas est parsemé de granules. Le diamètre des granules augmente légèrement du stade 1 au stade 5 suite à une incorporation d'une faible quantité de substance.

La coloration des hépatopancréas varie en fonction des stades de maturité au sein d'une même espèce bien que Genten et al. (2011) aient démontré que le foie est de couleur brun rouge chez les carnivores et brun chez les herbivores.

L'étude histologique de l'hépatopancréas a révélé qu'il est un organe mixte constitué du foie et du pancréas. L'architecture histologique du foie des poissons étudiés est identique à travers les différents stades de maturité comme les travaux de Genten et al (2011) l'ont relevé. Alors que le foie des Mammifères est divisé en lobes qui sont ensuite subdivisés en lobules (Leeson et al., 1971; Hould, 1982), celui des Poissons en est dépourvu. Le foie des Poissons contient un parenchyme qui est constitué essentiellement d'hépatocytes. Le parenchyme hépatique renferme des centres mélanomacrophages à proximité de certains vaisseaux sanguins et canaux biliaires. Cette description corrobore celle de Díaz et al. (1999).

Les tissus hématopoïétiques ont été observés dans les parenchymes hépatiques étudiés confirmant ainsi les résultats deTavares-Dias et al. (2000) et Barbosa et al. (2011). 
La vascularisation s'intensifie avec le stade de maturité et présente une ramification accrue. Ce qui permet au foie de recevoir des nutriments issus de la digestion afin de les stocker. Ainsi le glucose est stocké sous forme de glycogène dans les hépatocytes: c'est la glycogénèse. El-Bakary et El-Gammal (2010) ont observé dans les hépatocytes de Sparus aurata du glycogène et des lipides.

Les cellules pancréatiques se différencient de manière centripète et autour des branches de veine porte. Elles migrent de la périphérie vers le centre de l'organe hépatique en se bifurquant. Bruslé et Anadon (1996) ont rapporté que le tissu pancréatique exocrine se développe autour de la veine porte pendant l'ontogenèse et peut rester extrahépatique ou pénétrer plus ou moins profondément dans le parenchyme du foie en fonction des espèces de poissons. Le pancréas exocrine est constitué par des amas de cellules pyramidales disposées en cordons ou en acini séreux. Les îlots de Langerhans constituent la partie endocrine du pancréas comme chez les Mammifères.

La structure histologique de l'hépatopancréas lui confère différentes fonctions. L'étude microscopique de l'hépatopancréas a révélé que le parenchyme hépatique est constitué d'hépatocytes capables d'accumuler des substances protéiques dans le milieu intra-hépatocytaire. Les auteurs tels que HOAR (1957) et BERTIN (1958) ont mentionné que chez les poissons, l'ovogenèse et la spermatogenèse nécessitent un apport important d'énergie qu'ils stockent essentiellement dans le foie sous forme de lipides. Bouhbouh (2002) a mis en exergue le lien entre le poids des foies des poissons et la maturation de leurs gonades. Selon ces auteurs, l'hépatopancréas constitue un organe de stockage de substances qui serviront au cours de la reproduction.

L'hépatopancréas intervient aussi dans la digestion. Selon Genten et al (2011), la bile est activement élaborée par les hépatocytes et sécrétée dans canalicules puis convoyée dans la vésicule biliaire où elle est stockée et concentrée. En fonction des besoins de la digestion, elle est libérée dans le canal cholédoque et est déversée dans le duodénum. Cette substance non enzymatique facilite leur hydrolyse par les lipases pancréatiques.

L'hépatopancréas est une glande dite amphicrine car produit des sécrétions de type endocrine et de type exocrine. Ceci est dû au fait que les hépatocytes qui le composent possèdent une double polarité : l'une vasculaire correspondant à la fonction endocrine et l'autre canaliculaire correspondant à la sécrétion biliaire (Coujard, et al., 1980; HouId, 1982). Quant aux îlots de Langerhans, en se référant aux travaux de Beccaria et al. (1990), il existe quatre types de cellules principales dans les îlots: les cellules- $\alpha$ : qui assurent une production de glucagon, les cellules-B produisant de l'insuline, les 
cellules-D produisant de la somatostatine et les cellules-PP qui sécrètent le polypeptide pancréatique.

L'hépatopancréas regorge des cellules qui assurent un rôle de défense. Chez les Poissons, ce sont les cellules mélanomacrophages et le tissu hématopoïétique qui assurent la tâche immunitaire Genten et al (2011). Selon ces auteurs, les mélanomacrophages phagocytent diverses substances étrangères et des déchets formant ainsi des phagosomes qui fusionnent avec des vacuoles d'enzymes lytiques ou lysosomes. La grande vacuole ainsi formé constitue le phagolysosome. Ces derniers s'agrègent pour former le centre mélanomacrophage qui semble donc fonctionner comme de véritables organes d'épurations de l'organisme. Ils jouent ainsi un rôle dans le mécanisme de la défense des poissons. Le nombre, la taille et le contenu en mélanine des centres mélanomacrophages peuvent varier en fonction du sexe, et ont tendance à augmenter avec l'âge ou lorsque les conditions de vie deviennent défavorables. Les mélanomacrophages ont plusieurs fonctions chez les Poissons, tels que le traitement de l'antigène dans la réponse immunitaire, la destruction, la détoxication ou le recyclage de matériaux endogènes et exogènes, des dépôts de métabolites de cellules mortes, $y$ compris les globules rouges, ainsi que la réaction à différents antigènes (Campos et al, 2008; Manrique et al, 2014).

\section{Conclusion}

L'hépatopancréas chez les Poissons présente des morphologies variables selon les espèces. Par ce caractère il diffère du foie des Mammifères qui est un organe de forme lobée. L'hépatopancréas des Poissons est un organe amphicrine constitué de deux entités, le foie et le pancréas. Cette organisation est différente de celle des Mammifères chez lesquels le foie et le pancréas sont deux organes distincts.

$\mathrm{Au}$ plan microscopique, 1'hépatopancréas rassemble toutes les structures observées au niveau du foie et du pancréas des Mammifères. L'hépatopancréas des Poissons contiennent des canaux biliaires, des vaisseaux sanguins, des centres mélanomacrophages, du tissu hématopoïétique, des hépatocytes, des acini séreux pancréatiques et des îlots de Langerhans. Ces différents éléments contribuent au stockage des substances qui seront ultérieurement utilisées dans la reproduction. De plus, il joue un rôle de défense par l'action des cellules mélanomacrophages. En tant que Vertébrés, les Poissons sont des organismes dont 1'hépatopancréas semble être à un stade inférieur de différenciation par rapport aux Mammifères. 


\section{Remerciements}

Toute notre gratitude à madame KONE FATOU EVE Proviseur du Lycée Alassane Ouattara d'Anyama. Nous exprimons nos sincères remerciements à monsieur KOFFI TANOH Technicien du GERME à la retraite, à monsieur COULIBALY FANDIAGA MARCEL professeur d'anglais au Lycée Alassane Ouattara d'Anyama et à monsieur SOUMAHORO IBRAHIMA, Doctorant au Laboratoire de Biochimie pour leur aimable collaboration.

\section{References:}

1. Barbosa M.C., Jatobá A., Vieira F.N . La culture de la graisse juvénile Snook

(Centropomus parallelus Poey, 1860) alimenté probiotique dans des conditions de laboratoire. Braz. Cambre. Biol. Technol. 54: 795-801. 2011.

2. Beccaria C., Diaz J.P., Gabrion J., Connes R. Maturation du pancréas endocrinien à la loup de mer, Dicentrarchus labrax L. (Teleostei): étude immunocitochimique et ultrastructurale. I. Cellules produisant du glucagon. Gen. Comp. Endocrinol., 78 (1): 80-92. 1990.

3. Bertin L ., Arambourg C. Systématique des Poissons, in:«Traité de Zoologie» publié sous La direction de P.P.GRASSÉ-MASSON et Cie, Edit., Paris, 13 (3) : Famille Serranidae 2388-2390. 1958.

4. Bertolucci B ., Vicentini C.A., Franceschini-Vicentini I.B., Bombonato. MTS Microscopie optique et ultrastructure du foie d'Astyanax altiparanae Garutti et Britski, 2000 (Teleostei, Characidae). Acta Sci. Biol. Sci., 30 (1): 73-6. 2008.

5. Bouhbouh S. Bio-ecologie de Barbus callensis (VALENCIENNE 1842) \& Barbus fritschi (GÜNTHER 1874) au niveau du reservoir Allal el Fassi (Maroc). These de doctorat national. Université sidi Mohamed Ben Abdallah, faculté des sciences dhar el mehraz fes : 167p. 2002.

6. Bruslé J., Anadon G.G . Structure et fonction du foie de poisson. Dans Munshi, JSD \& Dutta, HM (Eds.). Morphologie des poissons. Hollande-Septentrionale, Science Publishers 77-93. 1996.

7. Campos C.M., Moraes J.R.E., Moraes F.R . Histopatologia de fígado, bord e BACO de Piaractus mesopotamicus, Prochilodus lineatus e Pseudoplatystoma fasciatum parasitados por myxosporídios, capturados no Rio Aquidauana, Mato Grosso do Sul, au Brésil. Rev. Bras. Parasitol. Vétérinaire. 17: 200-2005. 2008.

8. Claudemir K.F., Renata A. C., Maria T. S. B., Carlos A. V., Irene B.F.V. Morphology and Histochemistry of the Liver of Carnivorous 
Fish Hemisorubim platyrhynchos. Int. J. Morphol 32(2):715-720, 20. 2014.

9. Chilima D. M. Strengthening the role of fish for nutrition security in Eastern and Southern Africa. Worldfish center, Lusaka office.27p. 2008

10. Coujard R. J. Poirier ., Racadot J. Précis d'histologie humaine. Les Presses de l'université Laval, Québec, pp. 482-490. 1980.

11. Díaz A.O., González C.M., García A.M., Devincenti C.V., Goldemberg. AL Caractérisation morphologique et histochimique du foie des poissons faibles striés, Cynoscion Guatucupa (Cuvier, 1830). Biocienc 7 (1): 67-78. 1999.

12. El-Bakary N.E.R.,El-Gammal H.L. Etudes comparatives histologiques, histochimiques et ultra-structurelles sur le foie de Mullet à tête plate (Mugil cephalus) et Sea Bream (Sparus aurata). Global Vet 4 (6): 548-53.2010

13. Genten F., Terwinghe E., Danguy A. Histologie illustrée du poisson. Editions Quae. 2011

14. Hoar W.S. Gonad and Reproduction, in : «Physiology of fishes N.Y. Brown ed. 1957.

15. Hould R. Histologie descriptive et éléments d'histopathologie. Décarie éditeurs. Montréal. P. 148- 151.1982.

16. Leeson, T.S. Et Leeson Cr 1971. Histologie. Masson et C" éditeurs. Paris. $451 \mathrm{p}$.

17. Manrique W.G., Claudiano G.S., Petrillo T.R., Pardi M.C., Pereira F.M.A., Belo M.A.A., Moraes J.R.E., Moraes F.R. Réponse des centres spléniques melanomacrophage de Oreochromis niloticus (Linnaeus, 1758) à des stimuli inflammatoires par le BCG et les corps étrangers. J. Appl. Ichtyol 30: 1-6. 2014.

18. Martoja R., Martoja Pierson M. Initiation aux techniques de l'histologie animale (Masson eds). Paris. 1967.

19. Paugy D., Lévêque $C$, Régimes alimentaires et réseaux trophiques. In : Lévêque $\mathrm{C}$ et Paugy D. (Eds.). Les poissons des eaux continentales africaines 156 p. 1999.

20. Tavares D.M., Martins M.L., Moraes F.R. Relação hepatossomática em peixes teleósteos de cultivo intensivo. Rev. Bras. Zool 17: 273281. 2000

21. Wallace R. A., Selman K., Cellular and dynamic aspects of oocyte growt,in teleosts.Amer. ZooL, $21: 325-343.1981$. 